\title{
Unmanned Driving Infringement Judgment Based on Wireless Sensor Network Data Mining
}

\author{
Menglu Yang \\ School of Law, Xi'an Jiaotong University, Xi'an, Shannxi, 710016, China \\ Correspondence should be addressed to Menglu Yang; menglu9393@stu.xjtu.edu.cn
}

Received 17 September 2021; Revised 8 October 2021; Accepted 9 October 2021; Published 2 November 2021

Academic Editor: Guolong Shi

Copyright (c) 2021 Menglu Yang. This is an open access article distributed under the Creative Commons Attribution License, which permits unrestricted use, distribution, and reproduction in any medium, provided the original work is properly cited.

\begin{abstract}
Based on the wireless sensor network unmanned driving infringement identification system, this paper focuses on the application of data mining technology and state machine technology and designs and implements a set of practical and effective. Self-driving cars can reduce the frequency of traffic accidents, alleviate urban traffic congestion, improve people's travel efficiency, and lower the threshold of driving and other social values. The data processing program and a number of algorithms are given, and a complete set of data processing procedures and algorithms are proposed, including the collection of raw sensor data, the preprocessing of the collected data, and the feature extraction of the processed data. In the experiment, the unmanned driving infringement monitoring network was first designed to conduct real-time monitoring of unmanned driving infringements during transportation and application. Aiming at the characteristics of unmanned driving infringements, a monitoring network platform was designed for remote control and large-scale monitoring. Secondly, according to the characteristics of the unmanned driving infringement monitoring sensor network, the unmanned driving infringement node monitoring terminal is designed. The monitoring terminal part mainly designs the sensor module, the wireless communication module, the display warning module power module, and the data mining processing module. The sensor modules, respectively, include temperature, humidity, and concentration sensors, and the communication mode in the communication module mainly adopts Wi-Fi. At the same time, the research is based on wireless sensor network, combined with data mining technology, puts forward a sensory data display system model based on data mining technology, and conducts an in-depth analysis of the sensory data display system model, including the logical level of the system, system architecture, and functional modules. Finally, it focuses on the specific application of data mining technology in environmental information analysis and prediction, uses JAVA programming and realizes a data analysis and display system based on wireless sensor network, and verifies the accuracy of the data mining algorithm. The experimental results analyze the application of data mining technology in the driverless infringement determination system and use a large number of unmanned driving infringements to analyze the determination rules, so as to realize the interaction between active people and driverless cars.
\end{abstract}

\section{Introduction}

Wireless sensor networks (WSNs) are composed of a large number of sensor nodes, each sensor node is a small microelectromechanical system, and they are randomly deployed in the monitoring area [1]. These sensor nodes are connected by wireless communication to form a multihop selforganizing network system. This network will collect and process the information of the observed object and send it to the monitoring terminal [2]. There are three elements in the composition of WSN, which are sensors, sensing objects, and observers. WSN is an emerging field of knowledge in recent years. It integrates sensor technology, microelectromechanical systems, wireless communication, and other technologies. The disciplines it involves are highly intersected and integrate modern hot research fields [3]. Compared with traditional motor vehicles, driverless cars realize intelligent driving, liberating human drivers from traditional motor vehicle driving. Driverless cars will be the main travel tool in the future. Through research, it is found that the traffic accident referred to in this article refers to the traffic accidents between unmanned vehicles and motor vehicles, 
nonmotor vehicles, and pedestrians in the automatic driving mode [4]. Therefore, the driverless car relies on the driverless system to make judgments and decisions during the operation process and no longer relies on the human driver to recognize and judge various situations during the operation like a human driver does. Then, the driverless car will have traffic. After the accident, the identification of fault has become a big problem [5].

The biggest difference between driverless cars and traditional motor vehicles is that the operators of the two are different. Traditional motor vehicles rely on human drivers to control the steering, speed, and braking of the car, while new driverless cars rely on no human driving system; unmanned driving system completely replaces the status of traditional human drivers and can complete most of the tasks of human drivers' running traditional cars [6]. After an unmanned vehicle traffic accident occurs, it becomes a difficult problem to determine the fault in the constitutive elements of the infringement liability of the traffic accident. In other words, how to determine the fault in the constitutive elements of the driver's liability and the infringement liability becomes a problem more difficult. The driverless car is a new type of car that runs on the driverless system [7]. Since driverless cars rely on driverless systems to operate, it poses a challenge to the traditional liability mechanism for tort liability for traffic accidents centered on human drivers. The traditional liability mechanism cannot continue to be used to identify driverless car traffic with liability for accident infringement, especially in terms of identifying the responsible subject and the composition of tort liability [8].

This paper is supported by data mining technology based on wireless sensor networks and found that driverless cars have characteristics compared with traditional motor vehicles. More importantly, it can find the core difference between driverless cars and traditional motor vehicles, which is conducive to discovery. The liability mechanism for tort liability for driverless car traffic accidents is different from the traditional liability mechanism for tort liability for motor vehicle road traffic accidents. The wireless sensor network monitoring terminal node passes the collected unmanned driving infringement nodes and surrounding environment parameters through $\mathrm{Wi}-\mathrm{Fi}$ is transmitted to the middle node, and the setting of the middle node is relatively close to the monitoring station to facilitate the supply of energy. The data is mined by the intermediate node and then sent to the monitoring center. In this paper, RBF data mining is mainly used for data fusion, and the RBF data mining algorithm is embedded in the intermediate node. The intermediate node performs data mining, outputs the accurate fusion result, and then forwards it to the monitoring center to reduce the energy consumed by data transmission. And through the form of the host computer interface, the real-time monitoring curve is displayed intuitively, and at the same time, the neural network algorithm is added to mine the historical data of the network to predict the trend of the network. It is helpful to discover the difference between the liability mechanism for tort liability for unmanned vehicle traffic accidents and the traditional liabil- ity mechanism for motor vehicle road traffic accidents and to focus on the differences between the two. It is convenient for monitoring personnel to predict some conditions in the unmanned driving infringement network as soon as possible, and the early warning mode is improved.

\section{Related Work}

The application prospects of sensor networks are very broad, and they can be widely used in military, environmental monitoring and forecasting, health care, smart homes, building condition monitoring, complex machinery monitoring, urban traffic, space exploration, large-scale workshop, and warehouse management, as well as airports, large-scale safety monitoring of industrial parks, and other fields. In the face of massive environmental data, data mining technology can analyze the collected environmental data in a highly automated manner, make inductive reasoning, and dig out potential patterns to analyze the environmental conditions and make predictions on the evolutionary conditions [9-11]. With the continuous development and improvement of sensor technology, wireless communication technology, and computing technology and with the in-depth research and wide application of wireless sensor networks, wireless sensor networks will gradually penetrate into all areas of human life.

Pearah [12] used microclimate sensors and video sensor network technology to complete a small-scale ecological environment monitoring, through limited sensor nodes and simple communication software for long-term data collection, aiming to study the impact of the microclimate in the bird's nest on birds, and also studied the automatic classification and real-time image data collection in the remote bird's nest and the embedded network sensing technology in the soil. Hildebrandt [13] uses the method of data mining to extract attributes related to intrusion detection by comparing the normal mode and the intrusion mode. There are 41 attributes in 4 types, which belong to several types, including character enumeration type (such as network protocol), binary type, continuous natural number type, and continuous integer type. The value range of the attribute is also different. Scharf [14] proposed several cluster-based anomaly detection methods. These methods have two common characteristics: (1) First, we use a special clustering algorithm to process the input data to obtain clusters and then to detect anomalies on the basis of. (2) It only needs to scan the data set several times, which is more efficient and suitable for large-scale data sets. Alfian et al. [15] pointed out that when there are data sets of different densities, $\mathrm{DB}(\mathrm{p}, \mathrm{d})$ anomalies often miss out part of the outlier data. They further concluded that whether the data is an outlier depends not only on the distance between it and the surrounding data, but also on the density in the neighborhood. The density in the neighborhood of an object can be described by the radius of the neighborhood containing a fixed number of nodes or the number of nodes contained in the neighborhood of a specified radius.

Due to the high degree of autonomy of unmanned vehicles and the absence of drivers, the traffic accidents of 


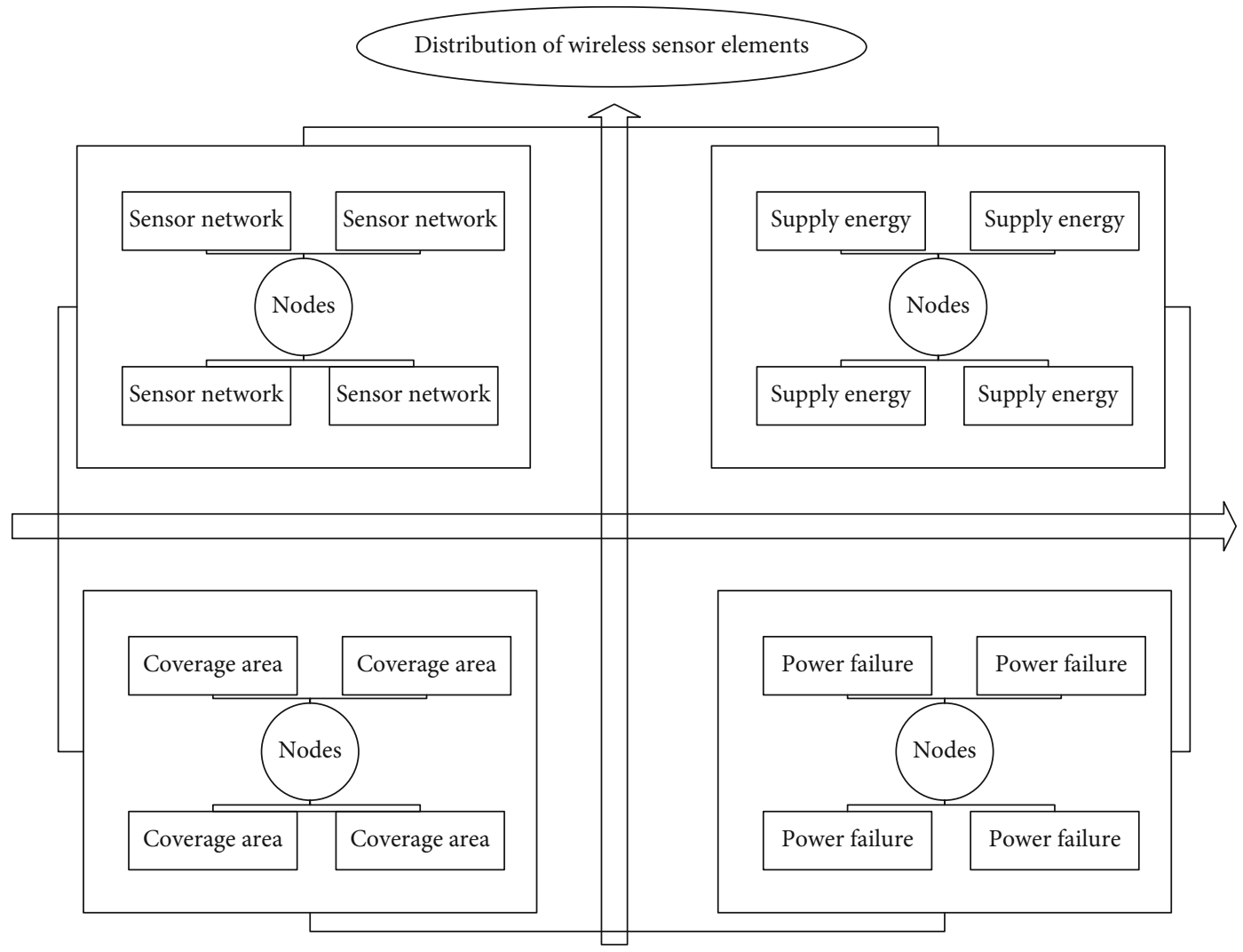

FIGURE 1: Wireless sensor distribution topology.

unmanned vehicles will have a certain impact on the current laws, and the responsible party cannot be determined. Some scholars believe that the unmanned car is proposed to be a special legal subject, and the unmanned car independently assumes the corresponding responsibilities. Mohanty et al. [16] believe that it is premature and unreasonable for unmanned vehicles to become legal subjects. The core of driverless cars is the artificial intelligence system. Different scholars have different views on the legal status of artificial intelligence. Driverless cars are regarded as drivers hired by everyone, and driverless cars drive on the road in accordance with the owner's instructions. Once driverless cars violate traffic rules and cause a traffic accident, they should refer to the existing tort law, and employees should commit torts. The employer should be the main body responsible for tort liability [17]. Some scholars also believe that in the case of a traffic accident in an unmanned vehicle, the rules of liability for elevator infringement can be applied analogously, with the unmanned vehicle manufacturer as the main body responsible. Both driverless cars and elevators can transport users from one place to another, and neither can control the operation of driverless cars and elevators in the process from the departure place to the destination [18-20]. Therefore, once a driverless car is involved in a traffic accident, the rules of liability for elevator infringement can be applied analogously, and the manufacturer of the driverless car is identified as the public carrier, who will be responsible for the tort liability for the driverless car traffic accident [21].

\section{Construction of an Unmanned Driving Infringement Judgment Model Based on Wireless Sensor Network Data Mining}

3.1. Distribution of Wireless Sensor Elements. Sensor networks usually have a large number of nodes, most nodes are in a static state, and the distribution of nodes is also very dense. Since nodes are mostly in the wild and distributed randomly, it is very difficult to supply energy. Therefore, sensor networks are mostly constrained by energy, and the node distribution is complex, resulting in nodes. Problems such as power failure or damage cannot be solved in time. Figure 1 shows the wireless sensor distribution topology.

The wireless sensor network is composed of a large number of cheap microsensor nodes deployed in the monitoring area. It is a multihop self-organizing network system formed by wireless communication. Its purpose is to cooperatively sense, collect, and process the sensing objects in the network coverage area. Information is send to the observer.

$$
X[t]=\{t \in R \mid x(1), x(2), \cdots, x(t)\} .
$$

A sensor network composed of a large number of tiny sensor nodes distributed in the environment or on objects can detect changes in these physical parameters caused by changes in user actions, thereby identifying user actions and monitoring of the environment, and according to the environment and 
the user's contextual information autonomously completes the specified tasks, realizing true environmental intelligence.

$$
y(n)=A \times X(n)-t \times x(n-1) .
$$

The perception layer node is the most basic unit in the entire unmanned driving infringement sensor network and the core of the function realization. It is mainly responsible for the collection of network information, determines the detection accuracy of the entire system, and is also the most important part of the system.

$$
f(x+1)-f(x)=\partial \frac{y(x, n)}{\|x(n)\|^{2}} \times x(n) .
$$

The sensing node is divided into 5 modules, a sensor module, a data processing module, a wireless communication module, a display alarm module, and a power supply module. However, in terms of network functions, each sensor node is a miniature embedded system, and each node also has a routing function. It can not only collect information, but also forward information and complete tasks with other nodes.

$$
g(x, k)=\frac{1}{n} \times \sum_{i=1}^{n}(1-\alpha(x))(1-\beta(x)) \times x(t) .
$$

With the in-depth study of sensor networks, researchers have proposed protocol stacks on multiple sensor nodes. A protocol stack includes the physical layer, data link layer, network layer, transport layer, and application layer, which corresponds to the five-layer protocol of the Internet protocol stack.

$$
\left\{\begin{array}{l}
\phi\left(i_{1}\right)=\frac{\left(\phi^{+}(i)+\phi^{-}(i)\right)}{2}, \\
\phi\left(i_{2}\right)=\frac{\left(\phi^{+}(i)-\phi^{-}(i)\right)}{2} .
\end{array}\right.
$$

In addition, the protocol stack also includes an energy management platform, a mobile management platform, and a task management platform. These management platforms enable sensor nodes to work together in an energy-efficient manner, forward data in the sensor network where the nodes move, and support multitasking and resource sharing.

3.2. Data Mining Algorithm Design. Data mining technology is to combine multiple sources of information through a certain method and process into a regular and simplified result. The hardware support of data mining is the sensor microelectromechanical system. The object of fusion is the multisource information collected by the sensor network. The core purpose of data mining is to coordinate, optimize, and comprehensively process the collected multisource information.

$$
\frac{\partial^{2} \alpha}{\partial x^{2}}+\frac{\partial^{2} \alpha}{\partial y^{2}}+\frac{\partial^{2} \alpha}{\partial z^{2}}-f(x)=0
$$

Data mining can also be defined in this way, the process of fusing various multiformat information sources collected by
TABLE 1: Description of sensor network nodes.

\begin{tabular}{lccc}
\hline $\begin{array}{l}\text { Node } \\
\text { number }\end{array}$ & $\begin{array}{c}\text { Operating } \\
\text { temperature } /{ }^{\circ} \mathrm{C}\end{array}$ & $\begin{array}{c}\text { Proportion } \\
\text { of nodes/\% }\end{array}$ & Weight \\
\hline 1 & 27.3 & 81.3 & 0.21 \\
2 & 24.1 & 78.2 & 0.24 \\
3 & 26.2 & 69.6 & 0.17 \\
4 & 25.4 & 66.4 & 0.38 \\
\hline
\end{tabular}

the sensor system together to generate various effective comprehensive information. The basic process is as follows: the front-end sensors of the system measure the temperature and humidity in the current measurement environment and the basic information of unmanned driving infringement and upload the measurement results of each sensor to the controller in real time through the transmission circuit. The controller converts the analog quantity into a digital quantity and then performs arithmetic processing on the obtained data. The data processing module processes the collected standard signals to obtain intuitive and simple data of concentration, temperature, and humidity. In the system, the alarm circuit is responsible for the alarm notification in the emergency state; the communication module is responsible for each sensor network node and the communication between each node and the central node: the power module includes two parts: the power supply module and the power management module, which are responsible for the power supply of the system and the power supply of the battery. Table 1 shows the description of sensor network nodes.

This article selects AM2301 digital temperature and humidity sensor to meet the temperature and humidity measurement requirements of the system. AM2301 is a digital output directly, which reduces the workload of the controller. It has the advantages of low price, good stability, high accuracy, and short feedback time. It can fully meet the requirements of the design for temperature and humidity measurement. When the MCU sends the start command, the terminal outputs the start level. After the sensor receives the start signal, it switches from the standby state to the working mode. For the total energy consumption, namely, the energy consumption of data collected by the sensor, the energy consumption of data processing by the microprocessor, and the energy consumption of radio transmission and reception, it is assumed that (1) different data query methods consume the same energy when the sensor collects data. (2) If the energy consumed by data processing is negligible, the energy consumed by the radio transceiver is the main factor that affects the total energy consumption of different data query methods. Therefore, the total energy consumption can be roughly measured according to the working time of the radio transceiver. After the sensor receives the start command, it will feedback the collected temperature and humidity information to the controller, and the controller will sort and decode the data fed back by the sensor to obtain the temperature and humidity information to participate in the calculation. The intermediate node of the sensor network is responsible for processing and forwarding the information collected by the sensing node. An 


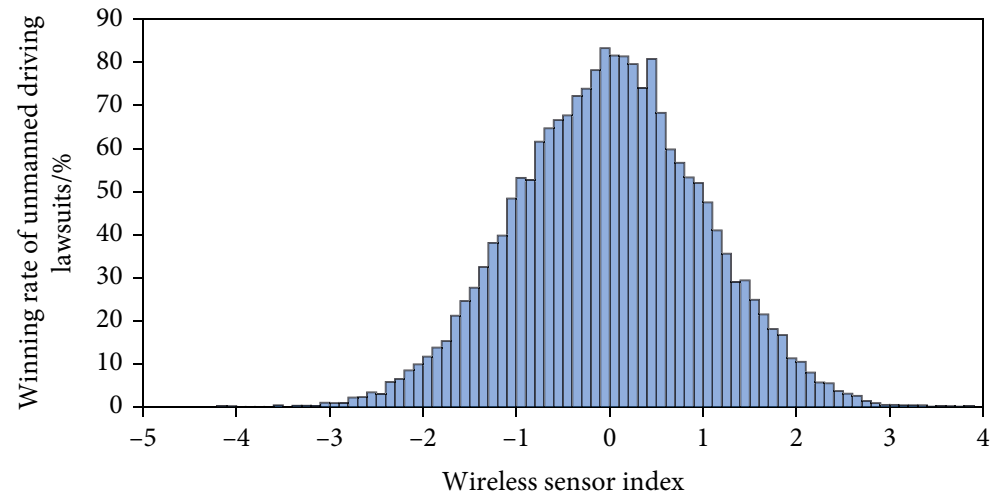

FIgURE 2: Statistical distribution of unmanned driving infringement evaluation winning rate.

intermediate node usually controls multiple sensing nodes. The middle node will merge the collected data and send it to the relay node, and the relay node will send it to the upper computer for monitoring. The real-time monitoring situation is intuitively displayed in the form of the host computer interface, and the prediction algorithm is calculated at the same time. First, it strengthens the prediction of the concentration trend in the network, which facilitates the monitoring personnel to predict the network status in advance and improve the early warning mode. The transmission circuit makes the fluctuation range of its output signal into a standard signal of $1 \mathrm{~V}$ to $3 \mathrm{~V}$ through two arithmetic processing of the input signal and completes the standardization of signal output.

\subsection{Unmanned Driving Infringement Judgment. The dam-} age in the constitutive elements of tort liability for driverless car traffic accidents is not much different from the damage in traditional motor vehicle road traffic accidents, and the rules for determining damage in traditional motor vehicle traffic accidents can be followed. How should the constituent elements such as illegal acts, faults, and causality in the infringement of unmanned vehicle traffic accidents be identified in different situations, and different imputation principles shall be applied according to the different subjects. The hybrid nature of the driving modes of unmanned vehicles determines whether unmanned vehicles are in automatic driving mode or manual driving mode when a traffic accident occurs, and the difference in their driving modes will also affect the type of responsibility. It can be seen that the traditional identification standards for the main body responsible for motor vehicle accidents are flawed, when identifying the responsible body of traffic accidents, the operation control theory and the operation benefit theory should be combined and comprehensively measured, that is, based on the operation control theory, emphasizing that the governor should bear the responsibility, adding operating benefits as a supplement under certain circumstances. Driverless cars are regarded as drivers hired by everyone, and driverless cars drive on the road in accordance with the owner's instructions. Once driverless cars violate traffic rules and cause a traffic accident, they should refer to the existing tort law, and employees should commit torts. The employer should be the main body responsible for tort liability.

Therefore, the traditional identification rules of the responsible body for road traffic accidents of motor vehicles cannot continue to be used to identify the responsible body for unmanned vehicle traffic accidents. In short, driverless car accidents have broken the traditional identification standards of the main body responsible for road accidents of motor vehicles. Before analyzing the constituent elements of unmanned vehicle infringement liability, it is necessary to judge the liability attribute of unmanned vehicle infringement first, because different liability attributes have different constituent elements of infringement liability, and different liability subjects, imputation principles, and even different liability attributes also affect the burden of proof and the plaintiff's winning rate. Figure 2 shows the statistical distribution of the winning rate of unmanned driving infringement evaluation. Although driverless cars have the intelligence that traditional cars do not have, in essence, driverless cars have not changed the attributes of their cars and still belong to the category of cars. Therefore, no one driving a car in a traffic accident should be attributed to the responsibility of the traffic accident. First of all, based on the theory of operation control, during the operation of an unmanned system car, it is the unmanned system that governs its operation. That is to say, the unmanned system replaces the status of a human driver, and the responsible subject should be an unmanned driving system. As a technology, an unmanned driving system can become the subject of responsibility like a human driver. Under the existing legal framework, the unmanned driving system obviously cannot become an unmanned system. In principle, the main body responsible for human-driving car traffic accidents does not work.

3.4. Model Data Factor Fusion. The ports CON1 to CON3 of the wireless communication module have built-in pull-up resistors inside the chip and can be directly connected to the controller. According to the chip manual, the CON3 terminal is left floating. The power supply current required by the chip when working in low power consumption mode is even as low as $0.1 \mathrm{~A}$, so that the wireless sensor network has better environmental adaptability. In order for the 


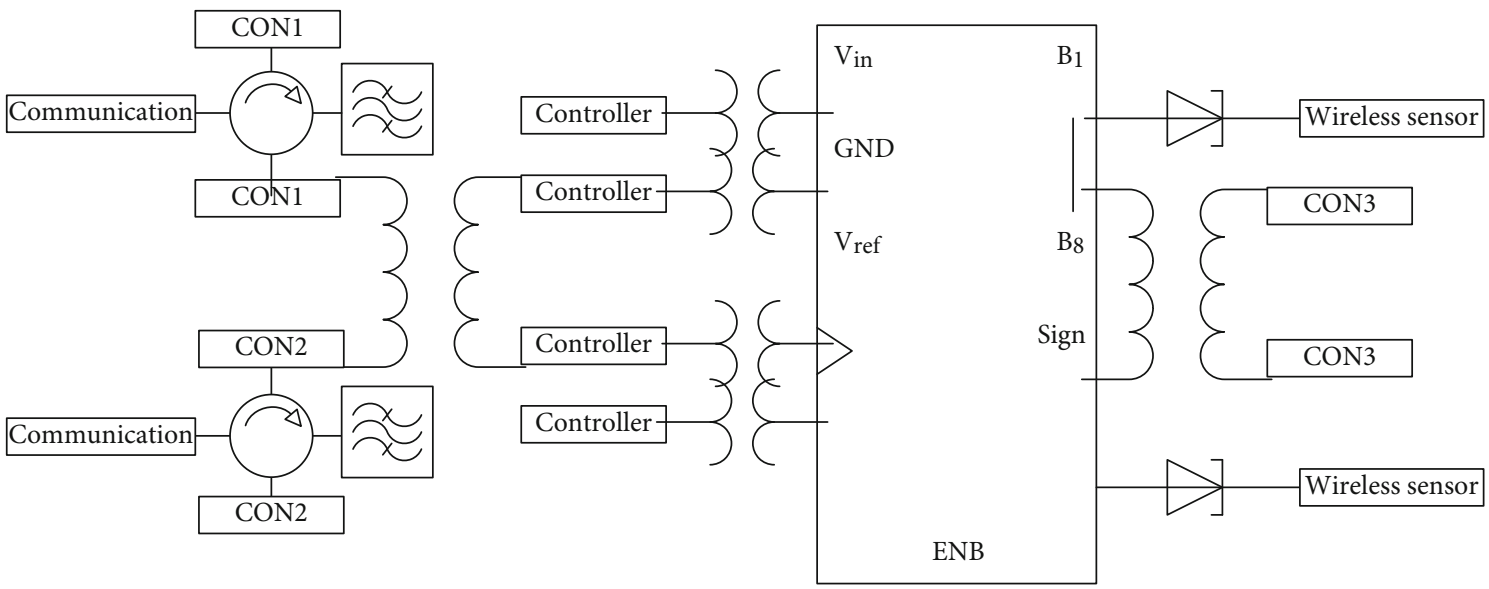

FIGURE 3: Circuit layout of wireless sensor network communication module.

communication module to operate normally, the following settings are required. After the chip is powered on, the default working mode is the normal mode, and the module can be switched from the normal mode to the low-power mode by continuously applying a low level for more than ten milliseconds to the sleep pin. Using the method of data mining, the sensor data can be analyzed offline or identified online. It can effectively solve the problem that the amount of data generated by sensors increases sharply after the increase in the number of sensors in the network. Data mining is the fusion of data at three different levels: original, feature, and decision-making levels, which can greatly improve the stability and robustness of the recognition system. Figure 3 shows the circuit layout of the wireless sensor network communication module. When both the controller and the communication module are in low power consumption, the communication between the nodes and the communication between the communication module and the controller will be stopped accordingly. When the controller is awakened from low power consumption, the communication module will be driven from low power consumption. After the power consumption changes to the normal mode, the system communication can return to normal.

The middle layer node is located in the center of the monitoring network, and each middle node controls a large number of sensor nodes. The sensing node passes the collected unmanned driving infringement nodes and surrounding environmental parameters through Wi-Fi communication method is transmitted to the relevant intermediate node, and the intermediate node performs data mining processing, selects suitable and representative data, and forwards these data to the relay node. The hardware structure of the intermediate node is similar to that of the perception layer node, and both have communication and alarm functions. But at the same time, the intermediate node is different from the perception node, and the intermediate node has the data mining function. In this algorithm, the radio transceivers of a small number of clustering central nodes are always in working state, while the radio transceivers of most noncentral nodes are in working state only during the following two times: (1) Each time, the system reclusters the nodes. At the same time, the node needs to exchange information with the system center node and the cluster center node; (2) when a single node finds that the sampling data has changed, it needs to send the data to the cluster center once. But in general, the time required for these two situations only accounts for a very small part of the system running time, which can be almost ignored. Therefore, compared with other methods, the method proposed by this algorithm can save the total energy consumption to the greatest extent. In the unmanned driving infringement sensor network, usually the intermediate node is relatively close to the monitoring station, and the man-machine exchange interface is designed for the intermediate node. When an alarm circuit prompts that there is an abnormality in the security of the network, or the perception layer node of the network is faulty, the man-machine exchange interface displays the specific location of the fault, and priority is given to transmitting this information to the monitoring base station.

\section{Application and Analysis of Driverless Infringement Judgment Model Based on Wireless Sensor Network Data Mining}

4.1. Wireless Sensor Data Filtering. Integrating the data volume, computing speed, and power consumption of the wireless sensor network design, this design selects a typical lowpower single-chip MSP430 as the controller of the hardware part of this article. The data processing capability of MSP430 during normal operation can fully meet the needs of this design, and its internal integrated $\mathrm{A} / \mathrm{D}$ converter saves the hardware equipment of the system and improves the costeffectiveness. What is more remarkable is the low power consumption of the single-chip microcomputer. The power supply current required by the chip when working in low power consumption mode is even as low as 0.1 , so that the wireless sensor network has better environmental adaptability. The power supply range can meet the conversion requirements of external power supply and battery power supply in this design at the same time. Its maximum output current limit parameter is $3 \mathrm{~A}$, with high conversion 


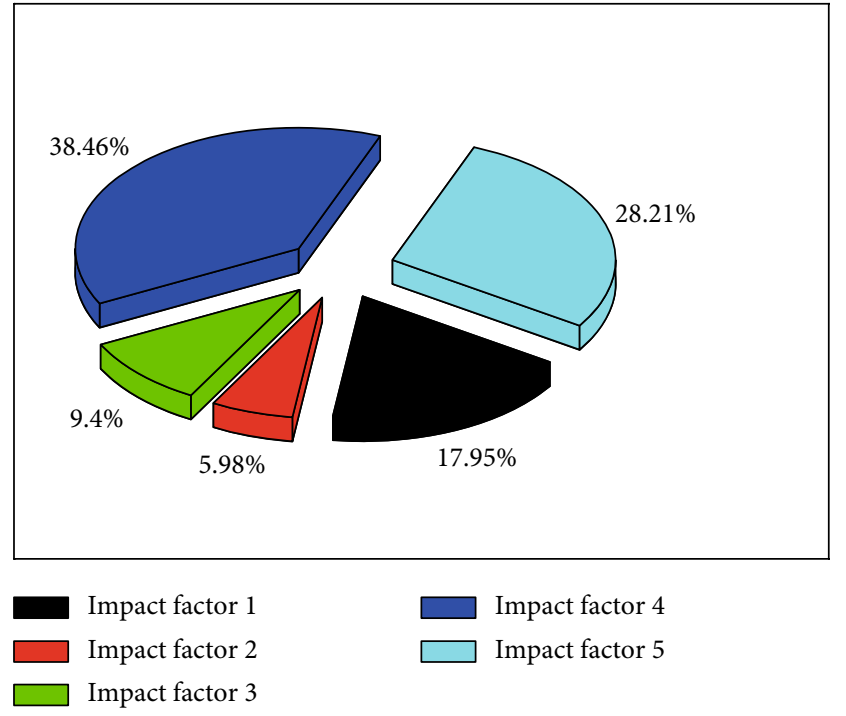

FIGURE 4: The fan-shaped distribution of the influence factors of extreme environment sensor ranging.

efficiency and good output characteristics. In order to ensure the ease of experimentation and the accuracy of experimental results, an experimental platform needs to be designed and built.

Figure 4 shows the fan-shaped distribution of the influence factors of extreme environment sensor ranging. First, the distance measurement experiment is carried out in a general environment as the control group of the extreme environment experiment. According to the experimental plan, the extreme environment sensor ranging experiment was carried out in sequence. The experimental result has the greatest influence on the sensor's attachment of large particles, and the wind speed and direction and fine dust have the least influence on its working stability. Since the attachment of large-particle objects on the surface of the lidar will directly lead to the lack of scanning data in some areas, it can be considered that the attachment of largeparticle objects to the environment has the greatest impact on the stability of the lidar range. By changing the number of iterations of the algorithm, a step-by-step approach to the target error can be achieved, thereby achieving the optimization of the training model. Secondly, according to the standard deviation distribution, it can be considered that the environmental factors that affect the stability of the sensor are temperature, dust concentration, and wind speed in descending order. After the unmanned vehicle receives the data collected by the on-board sensors, it transmits the information to the central processor and guides the vehicle to run or brake according to the processing results. The braking process of an unmanned vehicle mainly includes four stages, namely, the braking reaction stage, the braking coordination stage, the braking force growth stage, and the braking duration stage.

Experiments show that different environments have different effects on the stability of the sensor, but the ranging error is mostly within $1 \%$ of the ranging range, and it is impossible to specifically measure the impact of different environ-

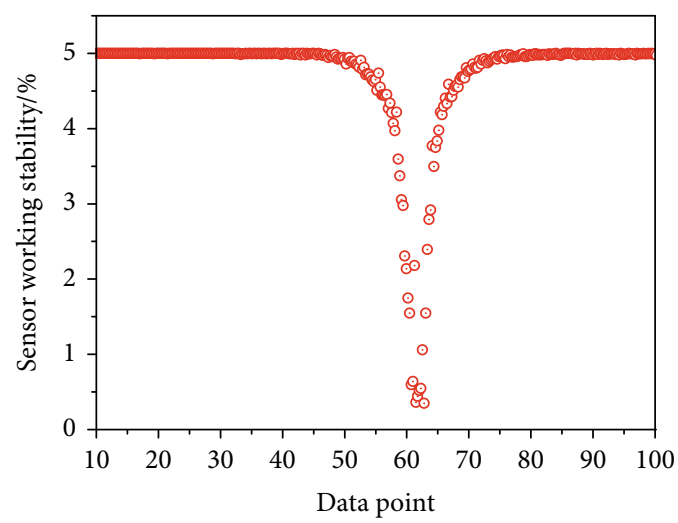

FIGURE 5: Sensor working stability curve.

mental factors on the stability of the sensor. Figure 5 shows the working stability curve of the sensor. Compared with the average value, the standard deviation can better reflect the degree of dispersion of a set of data. Therefore, in this study, the standard deviation of the error distribution is used as a working index to measure the stability of the sensor. Select the standard deviation of the ranging results in different experimental environments under a certain distance to get its distribution. It can be seen that the data mining clustering algorithm proposed in this chapter is significantly better than the original clustering algorithm in terms of running time for different data scales. The reason is that the original algorithm brings extra time cost to the discretization of the uncertain area of the data. Although the data mining algorithm is directly based on the probability distribution of the uncertain data in its uncertain area, it uses the R-tree index and probability. The threshold index PTI excludes most objects that do not meet the requirements in advance, thus improving the efficiency of the clustering process.

4.2. Data Mining Model Simulation Realization. The communication circuit in this design is mainly responsible for the data receiving and sending and signal transmission between sensor networks. This system uses Wi-Fi module EMW3180-4 is used as the control chip of the wireless communication module. It is a high-speed serial $13 \mathrm{Wi}$-Fi data transmission module, and it works in $2.4 \mathrm{G}$ ISM frequency band. It has TCP/IP protocol stack and Wi-Fi module driver at the same time. Through it, the wireless function of serial 13 devices can be realized. The current is less than $210 \mathrm{~mA}$ during operation and less than $1 \mathrm{~mA}$ during sleep. It has the advantages of low cost, fast speed, reliable transmission, and strong penetrating power. The parameter $s$ represents the rate of change of the value of the data item, and its upper limit is, unless otherwise specified, its default value is $5 \%$. Although the value is not large, because this chapter uses the time required for a data item in the wireless sensor network as the unit time and in the mobile computing environment, the time required for a data item in the wireless sensor network is very short. The change rate of the data item value is actually not small, which objectively reflects the uncertainty of the data. In the simulation experiment, the rate of change of each data item value is randomly distributed in 


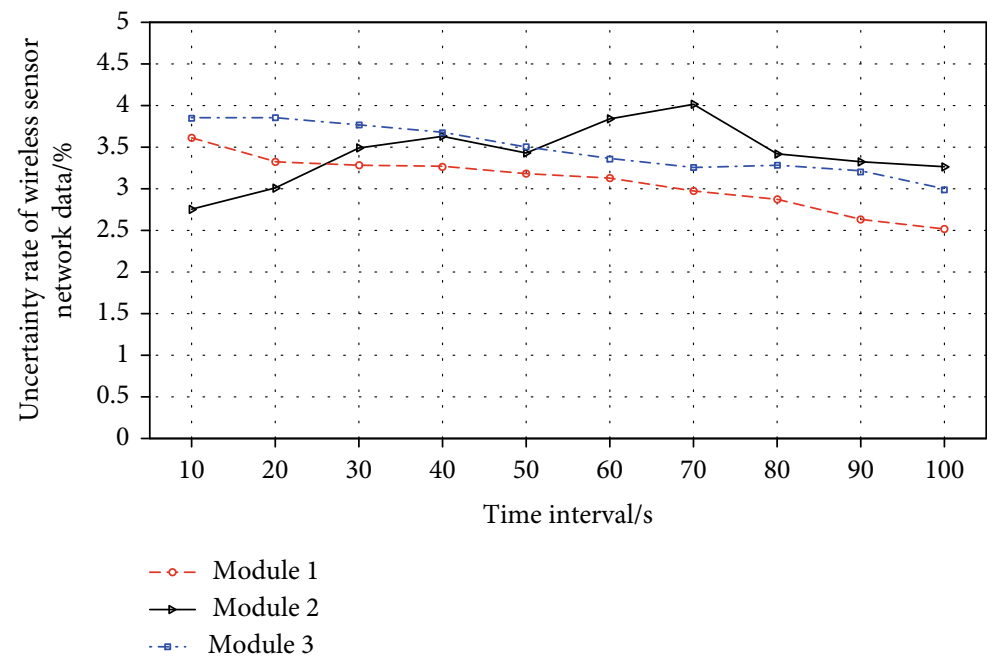

FiguRE 6: Line graph of uncertainty rate comparison of wireless sensor network data.

the range of $[0, s]$. The parameter $r$ represents the time interval for server-side data update. The value of $r$ will not affect the wireless sensor network scheduling process, but will only affect the absolute size of the data uncertainty rate. Without loss of generality, $r=100$ units are used in the experiment time.

If multiple network communication channels are available, data wireless sensor network scheduling on multiple channels can effectively reduce the data access delay. The simulation results of the total average access time corresponding to the number of different wireless sensor network channels are shown in the text. From this point of view, the increase in wireless sensor network channels can be seen as an increase in the bandwidth of the wireless sensor network. Figure 6 shows a line graph of the uncertainty ratio of wireless sensor network data. It can be seen that the UCDB-M method is more effective in improving the uncertainty rate of wireless sensor network data. Therefore, it can be said that the UCDB-M method sacrifices a small amount of access time in exchange for a significant reduction in data uncertainty, which is beneficial to improve the quality of queries based on these data. The use of multiple channels has little effect on the size of the average uncertainty rate of wireless sensor network data and its trend with $s$. The error information obtained by each unit can be used as the correction weight of each unit. Repeat the above two processes and wait for the predetermined target error range to be stopped. The reason for this phenomenon is that from the previous discussion on the scheduling strategy of multichannel wireless sensor networks, it can be seen that whether it is UCDB-M or VHM, the process of wireless sensor network scheduling is similar to that of single-channel wireless sensor network scheduling. The result (that is, the relative order in which each data item appears in the transmission) has nothing to do with whether multiple channels are used, and the total average uncertainty rate of wireless sensor network data is determined by the relative order in which each data item appears in the wireless sensor network. Therefore, the multichannel wireless sensor network has no advantages over the single-channel wireless sensor network in terms of the average uncertainty rate of the wireless sensor network data. From the perspective of wireless sensor network scheduling, multichannel wireless sensor network scheduling can be seen as distributing the results of single-channel wireless sensor network scheduling to multiple channels.

4.3. Example Application and Analysis. In order to analyze the performance of the clustering algorithm proposed in this chapter, relevant simulation experiments are carried out, and the test results are compared. The performance index of the comparison is the accuracy and efficiency of the clustering. The data set used in the simulation test comes from the geographic information benchmark data set. The value of the parameter is determined according to the proposed heuristic algorithm, and the parameter $p$ (probability threshold) is set to 0.8 . The simulation test was implemented with Visual C++ on a Pentium IV $2.4 \mathrm{GHz}, 512 \mathrm{MB}$ PC. We first use a training set and a test set (the attack type is the same as the training set) for experimentation. The anomaly determination criterion adopted in this chapter is that when the number of cluster elements in this category is less than $1 \%$ of the total data, it is determined that all elements in this category are anomalous points, that is, an intrusion point. The detection rate is the ratio of the number of attacks detected in the experiment to the actual number of attacks; the false detection rate is the ratio of the number of data detected as an attack but actually normal to the total number of normal data. Suppose the maximum moving distance of the space object from the last sampling to the current moment is $d$, and the value of $d$ reflects the uncertainty of the position of the moving object, the uncertainty interval of the spatial object is represented by a circle with the position of the spatial object obtained in the most recent sampling as the center and $d$ as the radius, and the position of the object in the uncertainty interval conforms to the normal distribution. For the data set containing the number of moving objects, $N=300$ in the experiment. Figure 7 shows the histogram of the sensor data detection rate of unmanned vehicles. 


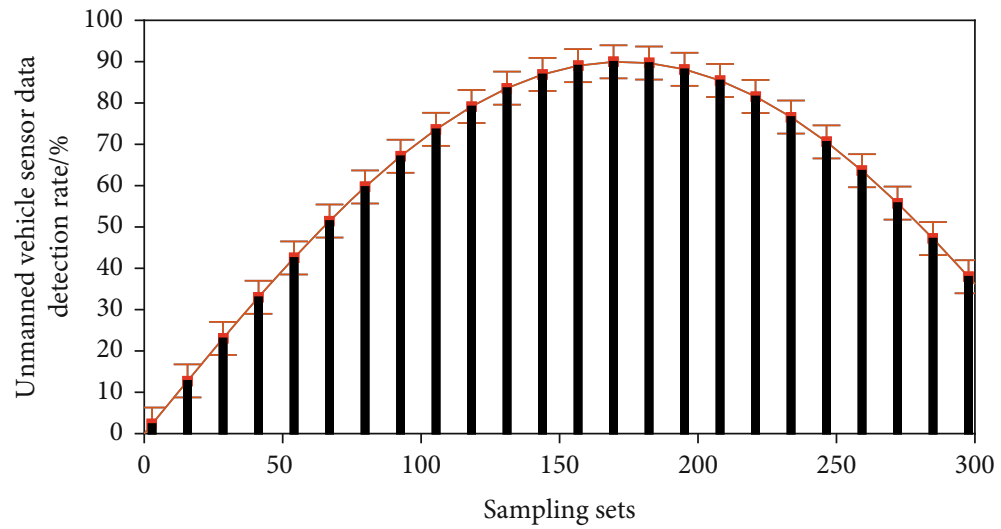

FIGURE 7: Columnar distribution of sensor data detection rate of unmanned vehicles.

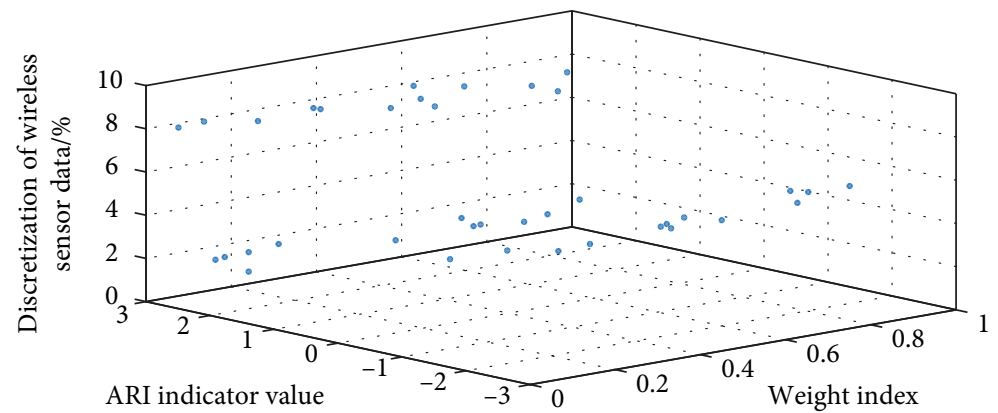

Figure 8: Discretization of three-dimensional scattered point distribution of wireless sensor data.

The working stability of the sensor of an unmanned vehicle will be affected to a certain extent, so the braking response time of an unmanned vehicle needs to consider the element of the reliability of the sensor. In this study, the vehicle speed and environmental factors in the braking reaction time are selected as the investigation objects, and the fuzzy logic control method is used to analyze the influence of the two on the reaction time, so as to obtain the safe distance between the environmental factors. The braking reaction time of the sensor is also different when the vehicle is running at different speeds. Among them, when the speed of the preceding vehicle is 0 , it means that the vehicle stops suddenly. The safety distance of an unmanned vehicle is a dynamic change process. When an unmanned vehicle is running at a certain speed, the greater the speed difference with the vehicle ahead, the greater the safe distance it maintains. When the vehicle speed is high, it is necessary to maintain a large following distance, and the braking reaction time is relatively prolonged. The working conditions corresponding to this fuzzy rule are as follows: when the vehicle speed is slow and the temperature and humidity of the environment where the sensor is located, the vehicle response time is medium. Since the fuzzy control system includes two input elements, the speed of the vehicle and the degree of influence of the environment on the stability of the sensor, the fuzzy set corresponds to four linguistic operators and six linguistic operators, so there are a total of 24 fuzzy rules. It can be found that the following vehicle only starts to brake after the preceding vehicle takes braking measures. When the vehicle model is constructed in the simulation process, since the selected models are the same, the speed curve slopes in parallel during the braking process. After the simulation, the distance between the two vehicles is $5 \mathrm{~m}$ greater than the safe parking distance, so the model verification is valid.

Since it is impossible to know the exact position of the moving object at the current moment in time, $D$ is actually impossible to obtain, and it only serves as a benchmark in the experiment. Figure 8 shows the discrete threedimensional distribution of wireless sensor data. The widely used Adjusted Rand Index (ARI) is used to compare the similarity of two clustering results. The larger the value of ARI is, the more similar the two clustering results are. It can be seen that as the value of $d$ increases, the error between the clustering results of the two algorithms and the ideal clustering of accurate data increases, indicating that the increase in data uncertainty causes the accuracy of clustering to decrease; for the same, the $d$ value of the clustering algorithm proposed in this paper is closer to the ideal actual result (larger ARI value) than the result of the original clustering algorithm, indicating that the data mining clustering algorithm is more effective. When the user's fault causes a traffic accident, there is no need to consider the manufacturer's product liability, and the user should be responsible for the general traffic accident. When an unmanned vehicle has a product defect that causes a traffic accident in an unmanned vehicle, the manufacturer shall bear the product liability. It can be seen that the data mining clustering algorithm proposed in this chapter is significantly better than the 
original clustering algorithm in terms of running time for different data scales. The reason is that the original algorithm brings extra time cost to the discretization of the uncertain area of the data. Although the data mining algorithm is directly based on the probability distribution of the uncertain data in its uncertain area, it uses the R-tree index and probability. The threshold index PTI excludes most objects that do not meet the requirements in advance, thus improving the efficiency of the clustering process. The reason is that the original clustering algorithm is calculated by sampling (discretization) of uncertain regions of the data, and the number of samples has a great influence on the calculation accuracy; but the data mining clustering algorithm proposed in this chapter does not have such a problem.

\section{Conclusion}

In this paper, data mining technology is mainly used to fuse the multinode data collected in the sensor network to reduce the energy loss caused by redundant data transmission. And we use data mining algorithms to predict, fusion, train, and simulate unmanned driving infringement monitoring data, so as to achieve the purpose of predicting network trends in advance. In this paper, a related wireless monitoring sensor network is designed based on the characteristics of unmanned driving infringement. The network is composed of the most basic sensing nodes, intermediate nodes, routing relay nodes, and upper computer monitoring interface. Among them, the sensing node is the smallest unit in the network. The sensing nodes are distributed in all corners of the monitoring environment. They are responsible for monitoring the surrounding environment parameters and transmit the collected parameters to the intermediate nodes. This article takes driverless car traffic accident tort liability as the research object and uses literature research method, data collection method, comparative analysis method, and comprehensive analysis method to compare and study traditional motor vehicle road traffic accident tort liability rules compared with driverless cars. The differences in the rules of tort liability are given for traffic accidents, to study the parts of driverless cars that break through the traditional rules of tort liability for motor vehicle traffic accidents, so as to put forward some of their own handling ideas and opinions, and hope to provide self-driving cars. Producers and system designers should be given other exemptions, and the scope of responsibility of producers and system designers should be reduced as much as possible. Producers and system designers should be encouraged to develop driverless cars with an active and open attitude. According to the characteristics of uncertain data, the concept of average data uncertainty rate is proposed, and then, an online broadcast scheduling strategy for uncertain data in a mobile computing environment is proposed, and simulation experiments are carried out for this strategy to prevent transmission errors and transmission errors. For performance testing under the conditions of error occurrence and multichannel wireless sensor network, the results confirm that the strategy proposed in this chapter can significantly reduce the data uncertainty by sacrificing a small amount of access time, which is conducive to the quality of query based on these data.

\section{Data Availability}

The data used to support the findings of this study are available from the corresponding author upon request.

\section{Conflicts of Interest}

The author declares no known competing financial interests or personal relationships that could have appeared to influence the work reported in this paper.

\section{References}

[1] I. Im, D. Shin, and J. Jeong, "Components for smart autonomous ship architecture based on intelligent information technology," Procedia computer science, vol. 134, pp. 91-98, 2018.

[2] Y. Sun, J. Liu, J. Wang, Y. Cao, and N. Kato, "When machine learning meets privacy in 6G: a survey," IEEE Communications Surveys \& Tutorials, vol. 22, no. 4, pp. 2694-2724, 2020.

[3] F. Jahan, W. Sun, Q. Niyaz, and M. Alam, "Security modeling of autonomous systems," ACM Computing Surveys (CSUR), vol. 52, no. 5, pp. 1-34, 2019.

[4] S. Clever, T. Crago, A. Polka, J. al-Jaroodi, and N. Mohamed, "Ethical analyses of smart city applications," Urban Science, vol. 2, no. 4, p. 96, 2018.

[5] M. Park, H. Oh, and K. Lee, "Security risk measurement for information leakage in IoT-based smart homes from a situational awareness perspective," Sensors, vol. 19, no. 9, p. 2148, 2019.

[6] P. K. Yu, "Data producer's right and the protection of machine-generated data," Tulane Law Review, vol. 93, p. 859, 2018.

[7] C. S. Chung, "Analysis on the possibility of electronic surveillance society in the intelligence information age," Journal of Platform Technology, vol. 6, no. 4, pp. 11-17, 2018.

[8] X. Wen, "Technology foresight research of industrial robot based on patent analysis," Journal of Data Analysis and Information Processing, vol. 7, no. 2, pp. 74-90, 2019.

[9] J. Kim and C. Lee, "Novelty-focused weak signal detection in futuristic data: assessing the rarity and paradigm unrelatedness of signals," Technological Forecasting and Social Change, vol. 120, pp. 59-76, 2017.

[10] R. Silva and R. Iqbal, "Ethical implications of social internet of vehicles systems," IEEE Internet of Things Journal, vol. 6, no. 1, pp. 517-531, 2019.

[11] R. A. Mashelkar, "Exponential technology, industry 4.0 and future of jobs in India," Review of Market Integration, vol. 10, no. 2, pp. 138-157, 2018.

[12] P. J. Pearah, "Opening the door to self-driving cars: how will this change the rules of the road," Journal of High Technology Law, vol. 18, p. 38, 2017.

[13] M. Hildebrandt, "New animism in policing: re-animating the rule of law," The sage handbook of global policing, pp. 406428, 2016.

[14] R. L. Scharf, "Game of drones: rolling the dice with unmanned aerial vehicles and privacy," Utah Law Review, p. 457, 2017.

[15] G. Alfian, J. Rhee, H. Ahn et al., "Integration of RFID, wireless sensor networks, and data mining in an e-pedigree food 
traceability system," Journal of Food Engineering, vol. 212, pp. 65-75, 2017.

[16] S. N. Mohanty, E. L. Lydia, M. Elhoseny, M. M. G. al Otaibi, and K. Shankar, "Deep learning with LSTM based distributed data mining model for energy efficient wireless sensor networks," Physical Communication, vol. 40, article 101097, 2020.

[17] A. G. Finogeev, D. S. Parygin, and A. A. Finogeev, "The convergence computing model for big sensor data mining and knowledge discovery," Human-centric Computing and Information Sciences, vol. 7, no. 1, pp. 14-16, 2017.

[18] L. Li, X. Li, Z. Lu, J. Lloret, and H. Song, "Sequential behavior pattern discovery with frequent episode mining and wireless sensor network," IEEE Communications Magazine, vol. 55, no. 6, pp. 205-211, 2017.

[19] S. Kumar and V. K. Chaurasiya, "A strategy for elimination of data redundancy in Internet of Things (IoT) based wireless sensor network (wsn)," IEEE Systems Journal, vol. 13, no. 2, pp. 1650-1657, 2019.

[20] G. M. Borkar, L. H. Patil, D. Dalgade, and A. Hutke, "A novel clustering approach and adaptive SVM classifier for intrusion detection in WSN: a data mining concept," Sustainable Computing: Informatics and Systems, vol. 23, pp. 120-135, 2019.

[21] H. Salehi, S. Das, S. Biswas, and R. Burgueño, "Data mining methodology employing artificial intelligence and a probabilistic approach for energy-efficient structural health monitoring with noisy and delayed signals," Expert Systems with Applications, vol. 135, pp. 259-272, 2019. 